\title{
Look better. Single atoms in chemistry, and single atoms in physics
}

\author{
Mario Pagliaro* \\ Dedicated to Professor Olimpia Lombardi, Universidad de Buenos Aires and Consejo Nacional de Investigaciones Científicas y \\ Técnicas, for all she has done to advance the fields of philosophy of chemistry and of physics
}

\begin{abstract}
Fostering fruitful collaboration between chemistry and physics scholars, the analysis of the differences in the practical approach to single atoms in chemistry and in physics affords a number of conceptual outcomes pointing to a more balanced relationship between chemistry and physics.
\end{abstract}

Keywords: single atom; chemistry foundations; chemistry methodology; Bose-Einstein condensate; chemistry and physics

\section{Introduction}

Mental visualization and association of chemical models for substances, especially molecular structures, atoms and electrons are the key mental activities founding chemistry's unique methodology amid natural sciences. ${ }^{[1]}$ Today, the concept is widely shared among scholars in chemistry education ${ }^{[2]}$ and foundations of chemistry. ${ }^{[3]}$

Drawing examples from different areas of chemistry, we have lately shown how visualization can be effectively used alongside with recent research outcomes and digital connectivity tools to enhance chemistry education with the aim to foster creativity in chemistry. ${ }^{[4]}$

Similar concepts are already used to improve chemistry education and enhance its attractiveness. For example, in some Switzerland's high schools "recent research work" is used to make chemistry "live, colourful and vivid to students, i.e. not something that has already been done by others before, but something that one can pursue oneself and that is totally new and original". ${ }^{\left[{ }^{[}\right]}$

Visualization of the chemical structure of organic molecules, metal complexes, biomolecules and even materials has been and continues to be the key research tool routinely used by chemists both as exploratory representation, ${ }^{[6]}$ and as a vehicle for communicating research results. ${ }^{[7]}$

Chemists are accustomed to the importance of the threedimensional structure of molecules since van't Hoff work with tetrahedral carbon and chirality published in 1875 in his seminal 43-page book La chimie dans l'espace. ${ }^{[8]}$

\footnotetext{
${ }^{*}$ Dr. M. Pagliaro

Istituto per lo Studio dei Materiali Nanostrutturati, CNR

via U. La Malfa 153

90146 Palermo (Italy)

E-mail: mario.pagliaro@cnr.it

Website: www.qualitas1998.net
}

Called by Kolbe as "totally devoid of any factual reality" and its author as "a transcendal chemist", ${ }^{[9]}$ van't Hoff was awarded the first Nobel prize in chemistry in 1901

As reminded by McBride in a highly recommended series of lectures in organic chemistry available online, ${ }^{[10]}$ van't Hoff not only ascribed the rotation of polarized light to optical isomers possessing stereogenic centers, but also predicted the existence of chiral allenes, a class of molecules that would not be observed for another 61 years.

A complete and elegant account on the emergence of the atomic and molecular structure theory in chemistry has been lately published by Rocke. ${ }^{[11]}$ In brief, the outcomes of van't Hoff's, Couper's, Cannizzaro's and Kekulé's work, is that "by the 1890s chemists had a far more sophisticated and powerful understanding of atoms and molecules than did physicists. The history of science literature, dominated by the physicists conception of atoms, has emphasized the debates over the existence of those particles, whereas chemists had recognized the heuristic value of the atomic theory long before". ${ }^{[12]}$

Commenting the famous Kekulé's dream of the benzene structure and reminding how Tesla, too, was said to be able to imagine the wear in his machines by simulating running them in his mind's eye,$^{[13]}$ the psychology and cognitive science scholar JohnsonLaird wrote in 1998 about "a rehabilitation of imagery in the face of the skeptics, but a limitation on imagery in the face of its more ardent adherents". ${ }^{[14]}$

From Kolbe in late $19^{\text {th }}$ century, to today's numerous physicists who assume that chemistry can (and should) be reduced to quantum physics (possibly derived from its "postulates"), the aforementioned skeptics comprise an extensive list. ${ }^{[15]}$

Studying Polanyi's paper on quantum chemistry, Bunge has shown as early as of 1982 that quantum chemistry, borrowing a central equation from chemical kinetics pre-dating quantum mechanics, does not follow from quantum mechanics alone. ${ }^{[16]}$ Yet, the argument continues to be repeated, with frequent calls for reduction of scientific fields according to Comte's $19^{\text {th }}$ century "hierarchy of the sciences" which would be reflected even by "bibliometric evidence". ${ }^{[17]}$

Fostering fruitful collaboration between chemistry and physics scholars, the subsequent analysis of the differences in the practical approach to single atoms in chemistry and in physics affords a number of conceptual outcomes pointing to a more balanced relationship between chemistry and physics. 


\section{An updated look at molecules}

As reminded by Feynman in one of his celebrated lecture series:

\begin{abstract}
«Early chemistry was very important for physics. The interaction between the two sciences was very great because the theory of atoms was substantiated to a large extent by experiments in chemistry. The theory of chemistry, i.e., of the reactions themselves, was summarized to a large extent in the periodic chart of Mendeleev, ... and it was the collection of rules as to which substance is combined with which, and how, that constituted inorganic chemistry. All these rules were ultimately explained in principle by quantum mechanics, so that theoretical chemistry is in fact physics». ${ }^{[18]}$
\end{abstract}

Actually, it has been the determination of the invisible chemical structure of organic compounds culminating in the work of van't Hoff republished in English in 1898 with the unequivocal title of The arrangement of atoms in space, ${ }^{[19]}$ that has led to the clear idea of molecules as ordered three-dimensional assemblies of atoms. In a powerful analogy between letters in words and atoms in molecules, these atoms are chemically bound to each other, Couper proposed in 1858 inventing the symbolic language to indicate how atoms are joined in molecules (Figure 1). ${ }^{[20]}$
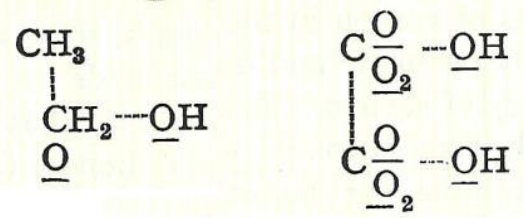

Figure 1. Archibald Couper's molecular structures, for alcohol and oxalic acid, using elemental symbols for atoms and lines for bonds (1858). [Reproduced from Ref.20]

Almost two centuries later, this idea is far from having lost its research and educational value. For example, McBride, a professor of organic chemistry renown for his excellence in teaching, has reassembled the modern teaching of organic chemistry as the answer to four main questions: ${ }^{[21]}$ l) How did we come to know what atoms are contained within molecules (composition)? ii) How did we come to know how atoms are connected to one another (constitution)? lii) How did we come to know the metric relationships within molecules (configuration)?, and $i v$ ) How did we learn to distinguish between left and right (conformation)?

Similarly, today chemists perform online calculations of continuous symmetry measure (a number between zero and 100 providing a quantitative description of the distance a particular structure has from perfect symmetry) ${ }^{[22]}$ improving their mental models of molecules, namely of molecular internal motion (vibration and rotation) and real molecular geometry including symmetry. ${ }^{[23]}$
"Now I am going to look at molecules in a different way. There is no sharp distinction between symmetry and no symmetry - there are a lot of levels in the middle"[23] commented in 2010 a chemistry high-school teacher after using the Molecular Symmetry Online online visualization tool to view molecules and their symmetry elements in three-dimensions.

Once again, profound innovation ${ }^{[22]}$ in structural theory of matter of practical relevance (continuous symmetry measures for instance are widely used in transition metal chemistry ${ }^{[24]}$ and in biochemistry) originated in 1992 from the work of a chemist (Avnir), working together with two computer science scholars (Peleg and Zabrodsky Hel-Or).

This single development in structural chemistry (symmetry as a continuous feature and mathematical variable) shows further evidence of the conceptual and practical value of what Lombardi and Labarca have correctly called the "autonomous existence of chemical entities". ${ }^{[25]}$

Indeed, Avnir and co-workers subsequently extended the CSM methodology originally conceived to treat the geometric symmetry of molecular structures defined as a set of points in threedimensional Euclidean space, to deal with the degree of symmetry of more complex mathematical objects commonly used in quantum chemistry such as wave functions, orbitals, and electron densities. ${ }^{[26]}$

In the words of Lombardi and Labarca:

"Which is the theory that informs us that orbitals do not exist? Quantum mechanics, of course. But why we do not ask molecular chemistry about the matter? What privilege does quantum mechanics carries for becoming the clue witness about what exists and does not exist in the world?

\footnotetext{
«There seems to be no other grounds for that privilege than an ontologically reductionistic attitude, according to which quantum mechanics is the best theory to describe the only "true" ontology: any description that disagrees with the quantum picture in unavoidably confined to a strictly nonreferring realm»[25]
}

In other words, only the rejection of the ontological reduction of chemistry to quantum physics can reverse an assumption that has become normal even among chemists.

The consequences are of the uttermost importance, starting from the need for chemists to properly understand for example that orbitals are states (i.e., mathematical constructs called wavefunctions), not entities, whose use in describing manyelectron atoms should be correctly seen as an approximation. ${ }^{[27]}$ 


\section{Single atoms in physics and in chemistry}

In 1995 Cornell, Wieman and co-workers reported the first experimental observation of a Bose-Einstein condensate, namely a dense collection of particles with integer spin (named "bosons" after Bose) condensing into the same quantum ground state. ${ }^{[28]}$

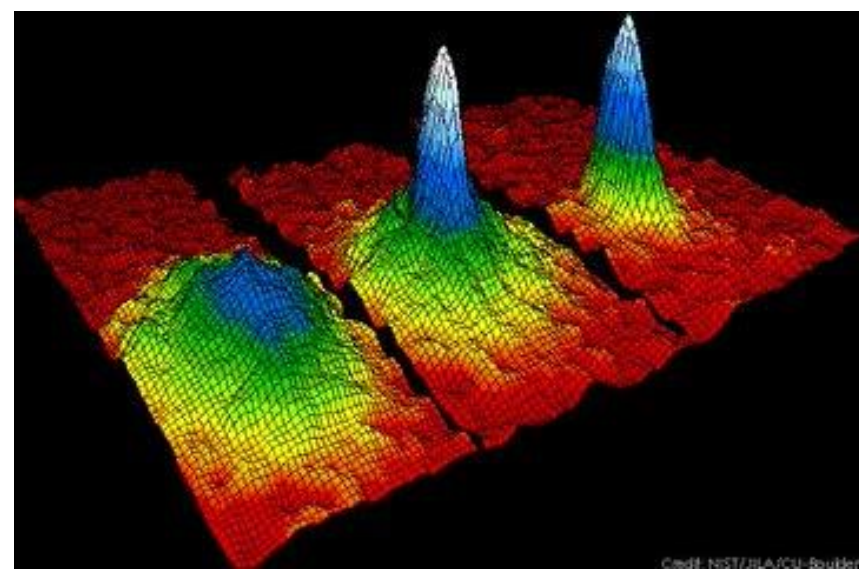

Figure 2. 3-D successive snap shots in time of velocity-distribution data of a gas of $87 \mathrm{Rb}$ atoms in which the atoms condense from less dense red, yellow and green areas into very dense blue to white areas. Left: just before the appearance of a Bose-Einstein condensate. Center: just after the appearance of the condensate. Right: after further evaporation, leaving a sample of nearly pure condensate. [Photograph of NIST/JILA/CU-Boulder, public domain]

Predicted in 1924-1925 by Bose ${ }^{[29]}$ and Einstein, ${ }^{[30]}$ the condensate was obtained by cooling a gas of ${ }^{87} \mathrm{Rb}$ atoms in gaseous state to $170 \mathrm{nK}$ affording a state of matter in which the single atoms, losing their individuality, behave like one large superatom, analogous to what happens with photons becoming indistinguishable in a laser beam". ${ }^{[31]}$

The discovery led to intense research activities focusing on the properties of ultracold atoms with implications for numerous application fields of condensed-matter physics, such as superfluidity, superconductivity, and magnetism. ${ }^{[31]}$

This single example shows how physicists think of single atoms: they are interested in controlling the quantum states and properties of single atoms, for example by identifying which mechanisms destroy the quantum properties of individual atoms by manipulating the magnetic state of a single $\mathrm{Fe}$ atom so as to avoid destructive interactions and improve the performance of magnetic quantum sensors consisting of a single atom. ${ }^{[32]}$

Chemists, on their turn, are interested in the chemical use of chemically and physically stabilized single atoms; for instance in the synthesis of desired chemicals, or in the decomposition of undesired chemicals.

For example, they devise methods to transform Pd nanoparticles deposited onto a metal organic framework made by zinc ions coordinated by four imidazolate rings (ZIF-8) into thermally stable supported $\mathrm{Pd}$ single atoms $\left(\mathrm{Pd}_{1} / \mathrm{ZIF}-8\right.$, Figure 3$)$ able to selectively catalyze the semi-hydrogenation of acetylene to ethylene, ${ }^{[33]}$ an important industrial process to purify the acetylene-contaminated ethylene feed for the production of polyethylene.

The Pd single atoms, now thermally and chemically stabilized, exhibit dramatically higher activity and selectivity than $\mathrm{Pd}$ nanoparticles due to the higher probability of molecular collision between $\mathrm{C}_{2} \mathrm{H}_{2}$ and $\mathrm{H}_{2}$ molecules on $\mathrm{Pd}_{1}-\mathrm{N}_{4}$ active sites, as a consequence of the preferential adsorption of $\mathrm{H}_{2}$ on the $\mathrm{N}$ site and $\mathrm{C}_{2} \mathrm{H}_{2}$ on the $\mathrm{Pd}$ site identified by quantum chemistry

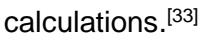

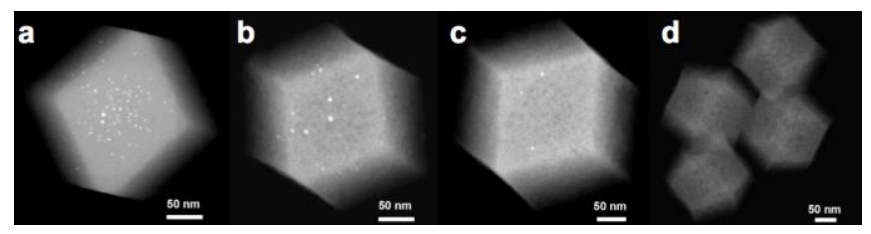

Figure 3. HAADF-STEM images of (a) Pd-nanoparticles@ZIF-8, (b) intermediate I (pyrolyzing Pd-NPs@ZIF-8 at $900^{\circ} \mathrm{C}$ for $30 \mathrm{~min}$ ), (c) intermediate II (pyrolyzing Pd-NPs@ZIF-8 at $900^{\circ} \mathrm{C}$ for $90 \mathrm{~min}$ ) and (d) Pd single atoms. [Image courtesy of Professor Yadong Li, Department of Chemistry, Tsinghua University].

Both physicists and chemists are interested in effective and reproducible methods to prepare single atoms; but whereas the former scientists will use advanced technology such as that required to bring atoms to ultralow temperatures of 170 nanokelvin (170 billionth of a degree above absolute zero) and to extract the information from the experimental observables, chemists are interested in developing scalable methods to support single atoms to be used as main components of newly prepared catalysts for the synthesis of known and still unknown substances, namely the main objective and the greatest success of chemistry.

\section{Physics tools serving chemistry purpose}

In a further demonstration of the practical scope of the autonomous chemical methodology, ${ }^{[1]}$ the practical development of research in single-atom catalysis ${ }^{[34]}$ vividly renders how chemists use physics-based tools and theories to achieve the useful visualization of matter typical of their powerful method based on visualization of chemical building blocks (atoms, molecules and electrons) and their reassociation via reaction mechanisms.

In late 2018, a team of Chinese scientists led by Wei and Yao at China's National Synchrotron Radiation Laboratory reported the structure and dynamic evolution of active sites in a single atom Co catalyst during the electrocatalytic hydrogen evolution reaction from water electrolysis in $1 \mathrm{M} \mathrm{KOH}$ alkaline electrolyte. ${ }^{[35]}$

Hydrogen evolution is the key process in alkaline water electrolysers increasingly used across the world to synthesize highly pure hydrogen on industrial scale using low cost $\mathrm{Ni}$ as electrocatalyst at both electrodes ${ }^{[36]}$ (and not costly Pt or Ir as often reported in too many scholarly research papers). 
The new electrocatalyst $\left(\mathrm{Co}_{1} / \mathrm{PCN}\right)$ is comprised of atomically dispersed cobalt ( 0.3 wt\%) immobilized by forming structurally uniform $\mathrm{Co}_{1}-\mathrm{N}_{4}$ moieties in the framework of phosphorized carbon nitride (PCN). ${ }^{[35]}$

The operando X-ray absorption fine structure (XAFS) measurements alongside with Fourier transform infrared spectroscopy (FTIR) and density functional theory (DFT) calculations led the team to distinguish the electronic and geometric structural changes occurring on the Co site, starting with the formation of the highly oxidized $\mathrm{HO}-\mathrm{CO}_{1}-\mathrm{N}_{2}$ moiety upon binding between initially isolated $\mathrm{Co}_{1}-\mathrm{N}_{4}$ sites with $\mathrm{OH}^{-}$in solution. [35]<smiles>CC1(C)CC1[18OH]</smiles><smiles>NN[C@@H](N)O</smiles><smiles>CC(C)C(C)ONC(N)O</smiles><smiles>N[14CH2]O[14NH2]</smiles><smiles>CC(C)(I)CO</smiles><smiles>[CH]O[C@@H](N)O</smiles>

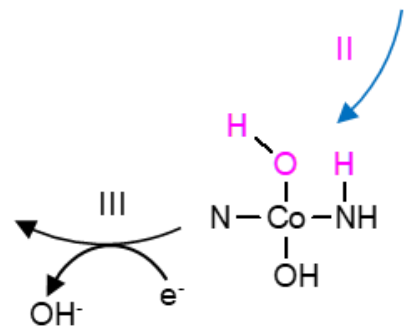

Figure 4. Hydrogen evolution reaction mechanism in alkaline electrolyte on C01/PCN. [Adapted from Ref.35].

What is relevant here is that eventually the team was able to propose a catalytic reaction mechanism for the alkaline hydrogen evolution reaction on the oxidized $\mathrm{HO}-\mathrm{CO}_{1}-\mathrm{N}_{2}$ moiety starting with $\mathrm{H}_{2} \mathrm{O}$ adsorption to form a $\mathrm{H}_{2} \mathrm{O}-\left(\mathrm{HO}-\mathrm{Co}_{1}-\mathrm{N}_{2}\right)$ reaction intermediate (Figure 4), which is fully analogous to the reaction mechanisms used by practitioners of research in organometallic chemistry.

In doing so, the powerful reaction mechanism approach typical of organic chemistry, ${ }^{[37]}$ which includes organometallic chemistry, extends to heterogeneous catalysis at the surface of single-atom catalysts.

"Powerful" here is synonymous of "fruitful", namely enabling chemists to devise practically important progress in understanding chemical reactions which translates either into new reaction paths to existing molecules, or to new molecules altogether.

Using the words of Lombardi and Labarca writing about the autonomous existence of chemical entities:

\begin{abstract}
«...molecular chemistry holds the winning card: its astonishing success in the manipulation of known substances and in the production of new substances is the best reason for accepting the existence of the entities populating its ontology. In other words, we are entitled to admit the reality of the molecular world on the basis of the impressive fruitfulness of molecular chemistry itself, independently of what physics has to say about the matter». ${ }^{25]}$
\end{abstract}

In brief, using the unique methodology of their science expanded by the use of incommensurable theories resulting from the interplay of quantum mechanics and heuristic chemical concepts (the numerous chemistry-derived concepts applied to quantum chemistry calculations), ${ }^{[1,16]}$ chemists across the world are ready to develop a variety of single-atom catalysts which truly hold the potential to revolutionize chemical manufacturing both in bulk and fine chemical industries, and clean electricity storage in solar hydrogen derived from water. ${ }^{[38]}$

\section{Conclusions}

A practical insight into the difference of conceiving single atoms in chemistry and in physics reveals the conceptual foundations on chemistry whose poor awareness amidst chemists themselves originates the "variety of concerns suggestive of some underlying uncertainties and self-doubts" reported by Heylin writing in 1998 about the need for chemistry to seek "a new contract with society". [39]

Whereas physicists look at single atoms as quantum entities seeking control of their quantum states and properties in sight of applications to fields such as electronics, photonics, superfluidity, superconductivity, and magnetism, chemists look at single atoms as chemical entities seeking control of their preparation and stabilization in sight of chemical applications chiefly in catalysis for chemical synthesis or environmental remediation, but also for chemical sensing. ${ }^{[40]}$

In doing so, chemists borrow from physics physical and conceptual tools such as transmission electron microscopes, synchrotron radiation and quantum mechanics theory, adapting them to their need to eventually visualize single atoms in the context of their powerful molecular structure and reaction mechanism approach through which they created the cornucopia of new, artificial substances benefiting society at large. ${ }^{[1,2,22,25]}$

Actually, as remarked by Lévy-Leblond, physics itself "despite its intrinsic mathematisation which seems to endow it with a more abstract than any other natural science, cannot be reduced to its mathematical formalism":[41]

«Formulas cannot be understood, neither can they be stated, for that matter, without words. The letters or other symbols that enter such formulas are but short-hand representatives of concepts, which have no existence independent of language. The words we use to name these concepts are of crucial importance as to their very grasping». ${ }^{[41]}$ 
Once again, also in the specific case of single atoms, there is not an hierarchical relation between physics and chemistry but rather a mutually beneficial relationship in which the strength of chemical theory and approach to matter that constitutes the core of its rich conceptual body complements the modern approach of physics to electrons in molecules and materials focusing on the ways individual particles interact with each other (for example in the presence of a magnetic field which led a team of physicists to lately discover a completely unexpected effect of the magnetic field on electronic properties of ferromagnetic material $\left.\mathrm{Fe}_{3} \mathrm{Sn}_{2}\right) .{ }^{[42]}$

Since visualization helps scientists in general "to envisage new possibilities by imagining certain spatial and physical properties and operations", ${ }^{[14]}$ it follows that by expanding and enhancing visualization abilities we can help scientists, and chemists in particular, to envisage more possibilities and creation of new substances and functional materials. ${ }^{[2,4,12]}$

Observing that "fruitful progress is often made at the fuzzy interface between disciplines; and because great discoveries were often associated with the ability of the researcher to look at a problem from an angle which is outside her own discipline" we concluded in 2010 that "collaboration with biologists, physicists, geologists etc. seeking the advice of chemists is and will increasingly be a feature common to leading chemical researchers". ${ }^{[1]}$

Accordingly, Rosenbloom and co-workers reported in 2015 a dramatic growth in knowledge production in chemistry between 1990 and 2009, which could not be explained by increasing financial expenditure but can be rather considered "a proxy for technological change", ${ }^{43]}$ and for information technologymediated change in particular, "given the coincidence of its timing with the spread of automatic laboratory data collection and analysis using personal computers and the internet". ${ }^{[43]}$

A decade later, and 30 years after physicists, research chemists started to use preprints ${ }^{[44]}$ to make rapidly and freely accessible on the internet the outcomes of their research, thereby enabling the numerous benefits of open science ${ }^{[45]}$ identified with the Budapest Open Access Initiative ${ }^{[46]}$ (2002) and the Hague Declaration $^{[47]}(2015)$.

Said benefits include, but are not limited to, improved population health, enhanced economic and social development, increased speed and progress of science, and enhanced new tools for education and research.

Chemists conceived the Li-ion battery (Akira Yoshino) and the hydrogen fuel cell (William Grove, likewise to Avogadro also a lawyer). Subsequently developed at industrial level with the aid of engineers (Bacon) and physicists, the former are the technologies enabling the transition from the internal combustion engine to electric vehicles. ${ }^{[48]}$

By the same token, showing evidence of lack of hierarchy of the sciences even from a practical viewpoint, it was a team of two physicists (Gerald Pearson and Daryl Chapin) and one chemist (Calvin Fuller) working together at an industrial company who conceived and developed in the early 1950s the silicon solar cell which 60 years later has enabled today's truly global boom of photovoltaic energy. ${ }^{[49]}$

As mankind strives to solve the related energy and environmental global crises, ${ }^{[50]}$ with chemists, physicists and engineers working together to advance the low cost clean electricity storage technologies urgently needed to achieve the transition to the solar economy, ${ }^{[51]}$ the time has come to look at the differences of perspective and methodology between chemistry and physics as a form of conceptual richness, and not as a matter of division and a barrier to fruitful collaboration among scholars working in different "departments" of the obsolete $20^{\text {th }}$ century academy organization.

\section{Acknowledgements}

Thanks to Professor Yadong $\mathrm{Li}$, Department of Chemistry, Tsinghua University, for kindly providing the high resolution HAADF-STEM images of the transformation of Pd nanoparticles to $\mathrm{Pd}$ single atoms.

\section{References}

[1] M. Pagliaro, On shapes, molecules and models: An insight into chemical methodology, Eur. J. Chem. 2010, 1, 276-281.

[2] L. L. Jones, R. M. Kelly, Visualization: The Key to Understanding Chemistry Concepts, In Sputnik to Smartphones: A Half-Century of Chemistry Education, M. V. Orna (Ed.), ACS Publishing, Washington (DC): 2015; pp. 121-140.

[3] J. A. Chamizo, A. Garritz, Historical Teaching of Atomic and Molecular Structure. In: M. Matthews M. (Ed.,) International Handbook of Research in History, Philosophy and Science Teaching, Springer, Dordrecht: 2014.

[4] M. Pagliaro, Chemistry education fostering creativity in the digital era, ChemRxiv 2018, 1. DOI: 10.26434/chemrxiv.7013009

[5] T. Rossel, M. Creus, La Chimie en Couleurs: Socially-Relevant and Original Research in Chemistry at High-Schools Using Modest Resources, ChemRxiv 2018, 1. DOI: 10.26434/chemrxiv.7396757

[6] R. Hoffmann, P. Laszlo, Representation in Chemistry, Angew. Chem. Int. Ed. Engl. 1991, 30, 1-16.

[7] M. Valle, Communication issues, handouts of the course Advanced Visualization for Chemistry, CSCS, Lugano, March 7-8, 2006. See at the URL: www.mariovalle.name/ChemViz/course/12-comm-issues.pdf

[8] J. H. van't Hoff, La chimie dans l'espace, P.M. Bazendijk, Rotterdam: 1875.

[9] A. J. Rocke, Kolbe Versus the "Transcendental Chemists": the Emergence of Classical Organic Chemistry, Ambix 1987, 34, 156-168.

[10] J. M. McBride, van't Hoff's Tetrahedral Carbon and Chirality, Lecture 26, Yale University, New Haven: 2008. See at the URL: https://oyc.yale.edu/chemistry/chem-125a/lecture-26

[11] A. J. Rocke, Image and Reality, University of Chicago Press, Chicago: 2010.

[12] P. J. Ramberg, Imagination in Chemistry, Science 2010, 329, 280-281.

[13] Cited in: R. N. Shepard, Externalization of mental images and the act of creation, In Visual learning, thinking, and communication, B. S. Randhawas and W. E. Corfman (Eds.), Academic Press, New York: 1978; pp. 133-189.

[14] P. N. Johnson-Laird, Imagery, Visualization, and Thinking, In Perception and Cognition at Century's End, J. Hochberg (Ed.), Academic Press, San Diego: 1998.

[15] J. van Brakel, Philosophy of Chemistry. Between the Manifest and the Scientific Image, Leuven University Press, Leuven: 2000. See in particular, chapter 5: The alleged reduction of chemistry. 
[16] M. Bunge, Is chemistry a branch of physics?, J. Gen. Philos. Sci. 1982, 13, 209-223.

[17] D. Fanelli, W. Glänzel, Bibliometric Evidence for a Hierarchy of the Sciences, PLoS One 2013, 8 (6): e66938.

[18] R. P. Feynman, R. Leighton, M. Sands, The Relation of Physics to Other Sciences, The Feynman Lectures on Physics, Addison-Wesley, Boston 1964; Volume 1, Chapter 3. See at the URL: www.feynmanlectures.caltech.edu/l_03.html.

[19] J. H. van't Hoff, The arrangement of atoms in space, Longmans, Green \& Co, London: 1898

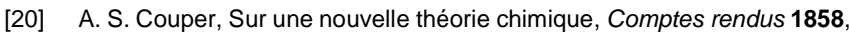
46, 1157-1160.

[21] M. D. Hollingsworth, J. A. Swift, B. Kahr, J. Michael McBride at 65 - An Appreciation, Cryst. Growth Des. 2005, 5, 2022-2035.

[22] H. Zabrodsky, S. Peleg, D. Avnir, Continuous symmetry measures, J. Am. Chem. Soc. 1992, 114, 7843-7851.

[23] I. Tuvi-Arad, R. Blonder, Continuous Symmetry \& Chemistry Teachers: Learning Advanced Chemistry Content through Novel Visualization Tools, Proceedings of the Chais conference on instructional technologies research 2010: Learning in the technological era, Y. Eshet-Alkalai, A. Caspi, S. Eden, N. Geri, Y. Yair (Eds.), The Open University of Israel, Raanana: 2011.

[24] S. Alvarez, P. Alemany, D. Avnir, Continuous Chirality Measures in Transition Metal Chemistry, Chem. Soc. Rev. 2005, 34, 313-326.

[25] O. Lombardi, M. Labarca, On the Autonomous Existence of Chemical Entities, Curr. Phys. Chem. 2011, 1, 69-75.

[26] P. Alemany, D. Casanova, S. Alvarez, C. Dryzun, D. Avnir, Continuous Symmetry Measures: A New Tool in Quantum Chemistry, In Reviews in Computational Chemistry, A. L. Parrill, K. B. Lipkowitz (Ed.s), Wiley, New York: 2017; pp. 289-352.

[27] P. Mulder, On the alleged non-existence of orbitals, Stud. Hist. Philos. Sci. B 2010, 41, 178-182.

[28] M. H. Anderson, J. R. Ensher, M. R. Matthews, C. E. Wieman, E. A. Cornell, Observation of Bose-Einstein Condensation in a Dilute Atomic Vapor, Science 1995, 269, 198-201.

[29] S. N. Bose, Plancks Gesetz und Lichtquantenhypothese, Z. Phys. 1924, 26, 178-181.

[30] A. Einstein, Quantentheorie des einatomigen idealen Gases, Ber. Akad. Wiss. Berlin 1925, 1: 3

[31] S. Ornes, Core Concept: How Bose-Einstein condensates keep revealing weird physics, Proc. Natl. Acad Sci. U.S.A. 2017, 114, 57665768.

[32] P. Willke, W. Paul, F. D. Natterer, K. Yang, Y. Bae, T. Choi, J. FernándezRossier, A. J. Heinrich, C. P. Lutz, Probing quantum coherence in singleatom electron spin resonance, Sci. Adv. 2018, 4: eaaq1543.

[33] S. Wei, A. Li, J.-C. Liu, Z. Li, W. Chen, Y. Gong, Q. Zhang, W.-C. Cheong, Y. Wang, L. Zheng, H. Xiao, C. Chen, D. Wang, Q. Peng, L. Gu, X. Han, J. Li, Y. Li, Direct observation of noble metal nanoparticles transforming to thermally stable single atoms, Nat. Nanotechnol. 2018, 13, 856-861.

[34] R. Ciriminna, M. Ghahremani, B. Karimi, R. Luque, M. Pagliaro, SingleAtom Catalysis: A Practically Viable Technology?, Preprints 2018 2018090069.

[35] L. Cao, Q. Luo, W. Liu, Y. Lin, X. Liu, Y. Cao, W. Zhang, Y. Wu, J. Yang, T. Yao, S. Wei, Identification of single-atom active sites in carbon-based cobalt catalysts during electrocatalytic hydrogen evolution, Nat. Catal. DOI: $10.1038 / s 41929-018-0203-5$.

[36] K. Zeng, D. Zhang, Recent Progress in Alkaline Water Electrolysis for Hydrogen Production and Applications, Prog. Energy Combust. Sci. 2010, 36, 307-326.

[37] W. Ogilvie, N. Ackroyd, C. Scott Browning, G. Deslongchamps, F. Lee, E. Sauer, Organic Chemistry Mechanistic Patterns, Nelson College, Toronto: 2018

[38] M. Pagliaro, Single-Atom Catalysis: A Forthcoming Revolution in Chemistry, Elsevier, Amsterdam: 2019.

[39] M. Heylin, The 'Central Science' Seeks A New Contract With Society, Chem. Eng. News 1998, 76, 2, 123-140.
[40] C. Zhao, H. Wu, A first-principles study on the interaction of biogas with noble metal (Rh, Pt, Pd) decorated nitrogen doped graphene as a gas sensor: A DFT study, Appl. Surf. Sci. 2018, 435, 1199-1212.

[41] J.-M. Lévy-Leblond, On the Plurality of (theoretical) Worlds, In Science as It Could Have Been: Discussing the Contingency/Inevitability Problem, L. Soler, E. Trizio, A. Pickering (Ed.s), University of Pittsburgh Press, Pittsburgh: 2015; pp. 335-358.

[42] J.-X. Yin, S. S. Zhang, H. Li, K. Jiang, G. Chang, B. Zhang, B. Lian, C Xiang, I. Belopolski, H. Zheng, T. A. Cochran, S.-Y. Xu, G. Bian, K. Liu, T.-R. Chang, H. Lin, Z.-Y. Lu, Z. Wang, S. Jia, W. Wang, M. Z. Hasan, Giant and anisotropic many-body spin-orbit tunability in a strongly correlated kagome magnet, Nature 2018, 562, 91-95.

[43] J. L. Rosenbloom, D. K. Ginther, T. Juhl, J. A. Heppert, The Effects of Research \& Development Funding on Scientific Productivity: Academic Chemistry, 1990-2009, PLOS ONE 2015, 10(9): e0138176.

[44] P. Demma Carà, R. Ciriminna, M. Pagliaro, Has the time come for preprints in chemistry?, ACS Omega 2017, 2, 7923-7928.

[45] S. Bartling, S. Friesike (Ed.s), Opening Science, Springer, Cham: 2014.

[46] Budapest Open Access Initiative, 14 February, 2002. See at the URL: budapestopenaccessinitiative.org

[47] The Hague Declaration on Knowledge Discovery in the Digital Age, Brussels, 6 May 2015. See at the URL: thehaguedeclaration.com.

[48] M. Pagliaro, F. Meneguzzo, The driving power of the electron, J. Phys. Energy 2019, 1, 011001.

[49] F. Meneguzzo, R. Ciriminna, L. Albanese, M. Pagliaro, The Great Solar Boom: A Global Perspective into the Far Reaching Impact of an Unexpected Energy Revolution, Energy Sci. Eng. 2015, 3, 499-509.

[50] F. Meneguzzo, R. Ciriminna, L. Albanese, M. Pagliaro, The energy population conundrum and its possible solution, arXiv 2016 arXiv: 1610.07298 [physics.soc-ph].

[51] B. K. Sovacool, How long will it take? Conceptualizing the temporal dynamics of energy transitions, Energy Res. Soc. Sci. 2016, 13, 202-215. 


\section{Look better.}

Single atoms in chemistry, and single atoms in physics

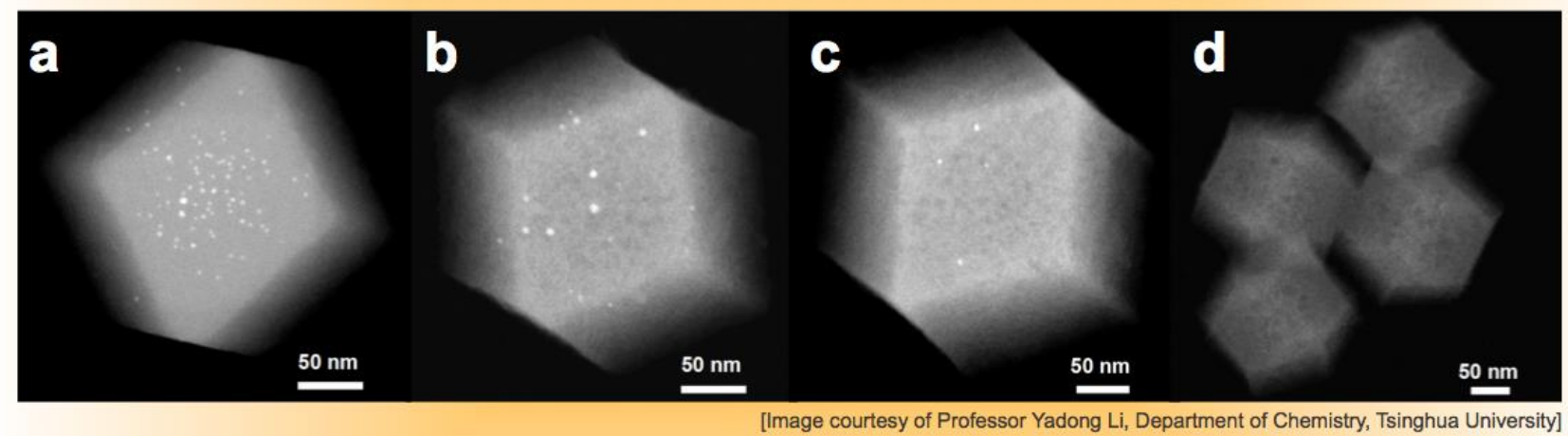

